\title{
Editorial
}

\section{Networking for Computer Games}

\author{
Jouni Smed \\ Department of Information Technology, University of Turku, 20014 Turku, Finland \\ Correspondence should be addressed to Jouni Smed, jouni.smed@utu.fi \\ Received 17 June 2008; Accepted 17 June 2008 \\ Copyright (c) 2008 Jouni Smed. This is an open access article distributed under the Creative Commons Attribution License, which \\ permits unrestricted use, distribution, and reproduction in any medium, provided the original work is properly cited.
}

Networking has become the main selling point for computer games: commercial games are expected to support multiplaying and the online game sites aim at supporting an ever increasing number of users. At the same time, new game console releases rely heavily on the appeal of online gaming, and a whole new branch of mobile entertainment has emerged with intention to develop distributed multiplayer games for wireless applications. This special issue on "Networking for computer games" focuses on the latest research done on networked computer games and presents five papers exploring different aspects of online multiplayer games.

Multiplayer computer games require both consistent and responsive networking. Consistency is important for maintaining a similar set of data for all players, whereas responsiveness requires that updates to the data are done as promptly as possible. These two requirements, however, are often contradictory and solving this consistencyresponsiveness dichotomy lies in the heart of real-time interactive networking. In the first paper "Towards an information model of consistency maintenance in distributed interactive applications," Xin Zhang et al. approach this topic by introducing a framework for analysing the state fidelity of predictive methods.

A massively multiplayer game can have tens of thousands simultaneous players from all of over the world, which means that the scalability of the chosen network architecture becomes critical. Moreover, a massive multiplayer game often requires maintaining a persistent game world, where the game progresses around the clock regardless whether a player takes part in it. The next two papers address this topic. In the second paper "High-level development of multiserver online games," Frank Glinka et al.describe a middleware system called real-time framework, which aims at raising the level of abstraction for the developer of an online game. In the third paper, "ALVIC versus the Internet: redesigning a networked virtual environment architecture," Peter Quax et al. present a generic framework for deploying a massive multiplayer online game using the existing Internet resources.

Mobile gaming and wireless games require a special attention to maintain a continuous and error-free flow information. In the fourth paper "The playing session: enhanced playability for mobile gamers in massive metaverses," Stefano Cacciaguerra and Gabriele D'Angelo introduce a mechanism, based on mimicking the player activities, which is capable of controlling the communication even in a case of a network failure.

The online game sites aim at providing the players more customized content according to the players preference and playing style. This requires methods for analysing the player behavior, which is also important in detecting players who cheat or otherwise misbehave in the game world. In the fifth paper "Visualization of online-game players based on their action behaviors," Keita lizuka and Ruck Thawonmas present an approach for recognizing different player-type clusters by visualizing the players in-game decisions.

Jouni Smed 

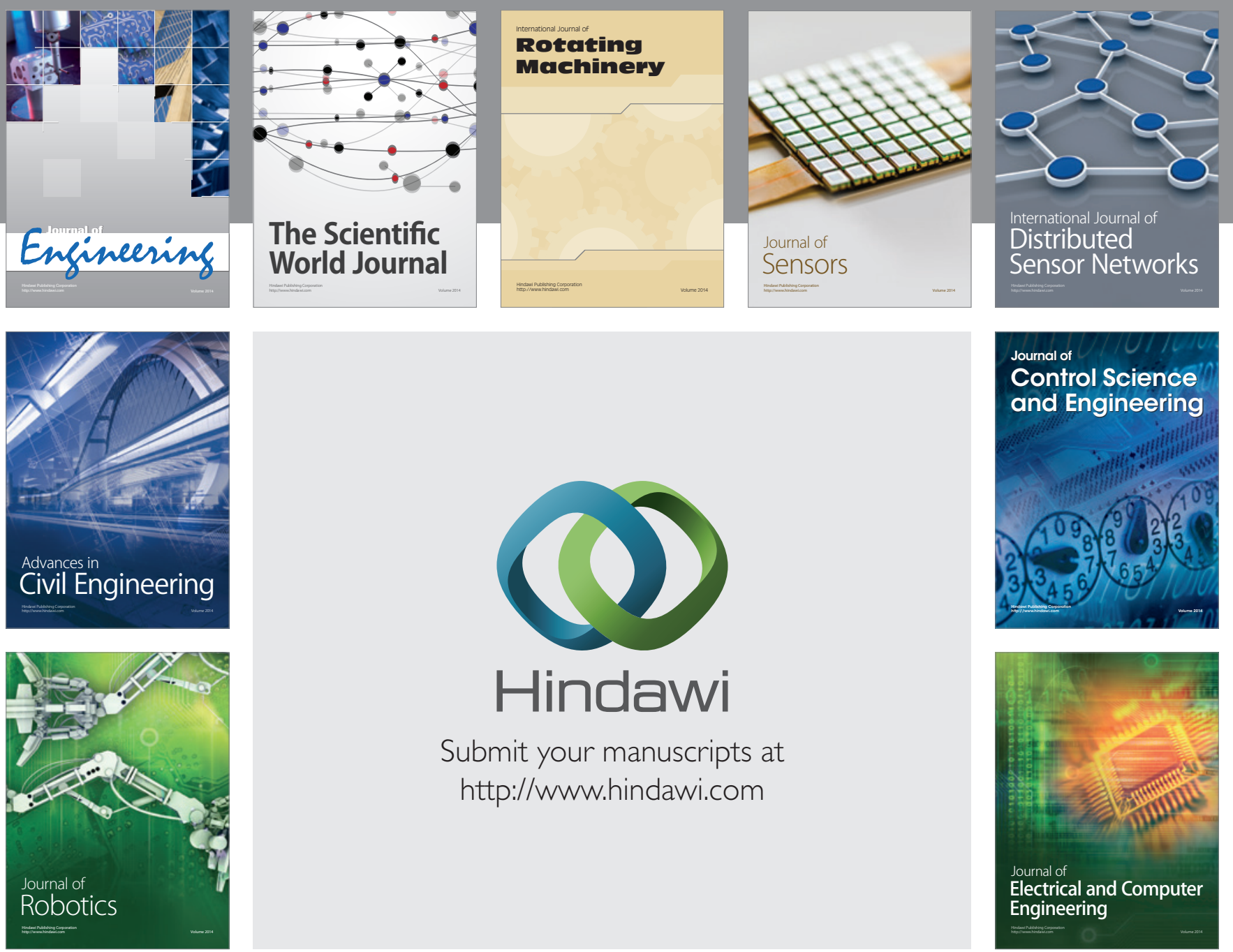

Submit your manuscripts at

http://www.hindawi.com
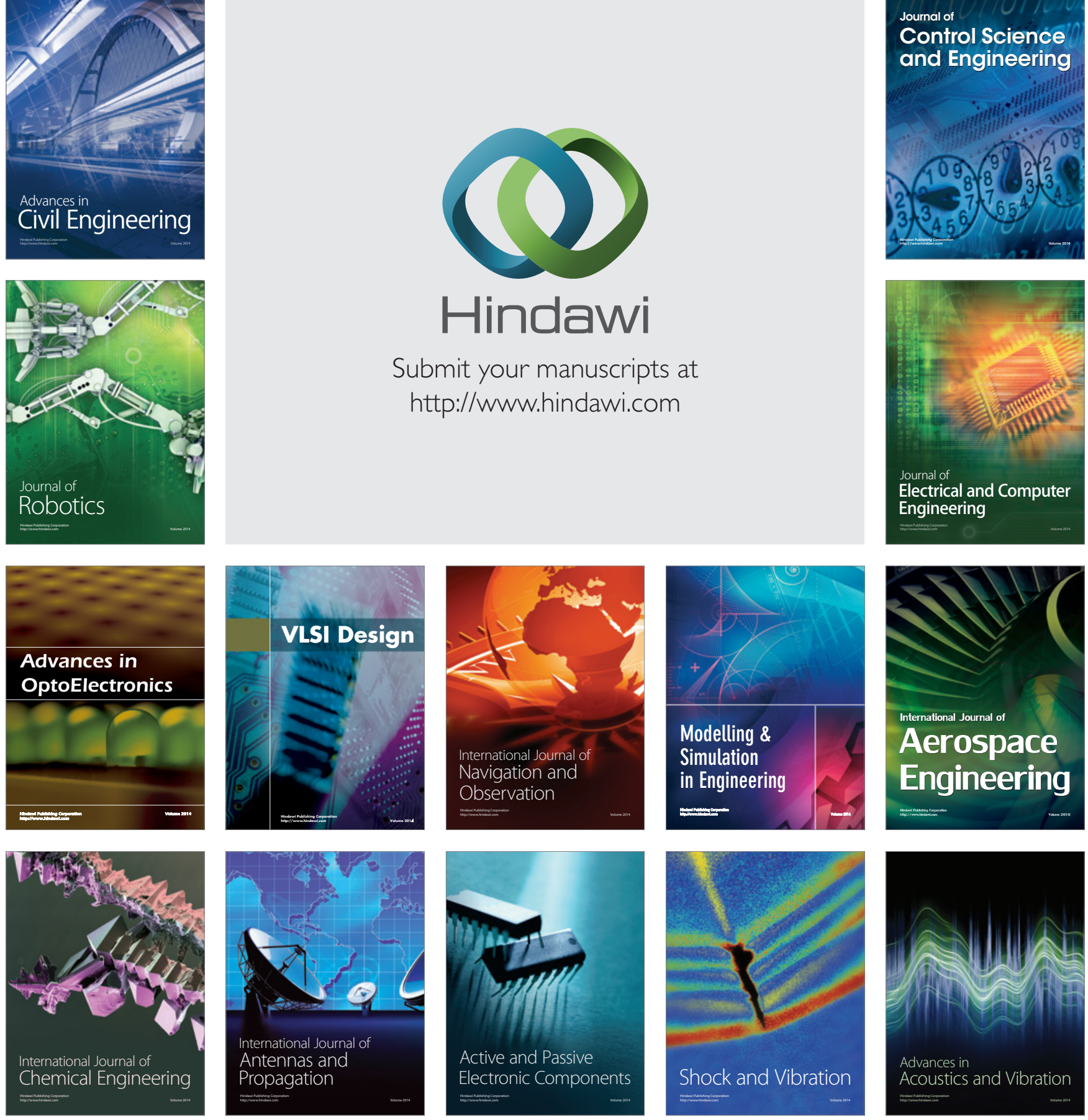